\title{
An improvement for dual channel sampling wattmeter
}

\author{
W.M.S. Wijesinghe ${ }^{1, \star}$ and Y.T. Park ${ }^{2}$ \\ 1 University of Science and Technology, Daejeon South Korea \\ ${ }^{2}$ Korea Research Institute of Standards and Science (KRISS), Electromagnetics Group, Daejeon South Korea
}

Received: 26 April 2010 / Accepted: 19 August 2010

\begin{abstract}
We present a digital sampling algorithm that uses two high resolution integrating voltmeters that are synchronized by a phase-lock loop clock to accurately measure the active and apparent power parameters for sinusoidal power measurements. The developed system provides high precision measurements of the root mean square, phase and the complex voltage ratio of the ac signal with sophisticated algorithm which process the data in frequency domain. The system was designed to be used at the Korea Research Institute of Standards and Science as reference power standard for electrical power calibrations. The detail uncertainty calculations have shown that the accuracy of the measurements were better than $4 \mu \mathrm{W} / \mathrm{VA}$ (for $k=1$ ) and the level of uncertainty was valid for the power factor range $0 \sim 1$ for both lead and lag conditions. The system is fully automated and allows power measurements and calibration of high precision wattmeters and power calibrators at the main power frequencies of 50 and $60 \mathrm{~Hz}$.
\end{abstract}

Keywords: Electrical power; sampling wattmeter; uncertainty calculation; digital signal processing; traceability

\section{Introduction}

Precise power measurement is very important in the area of energy maintenance to reduce power losses that directly affect the economy of all countries. Industries in all areas try to minimize power losses, while maintaining their traceability of power measurement. Therefore, the capabilities of power testing and calibration have grown and the demands on national metrology institutes (NMIs) to provide such services have increased. In order to respond to changing needs of the power measurement industries, Korea Research Institute of Standards and Science (KRISS) has to improve the level of uncertainty in electrical power measurements. The important factors of the power standards are determination of active power, apparent power and the power factor that minimizes the reactive power; the errors of these factors should be minimized for the power standard and its precise measurement.

The existing traceability maintenance system at KRISS is based on the power comparator method using thermal converters (TC) that have a high level of uncertainties. Therefore, the power standards laboratory of KRISS began research and development work of a sampling-based reference power standard to fulfill the requirement. The analog-to-digital converters (ADCs) are more important when a sampling technique is being employed (more details can be found in [1] and [2]). The sampling technique, dual channel method successfully used in [1] and [2] and single channel method was used in [3] and [4], was employed by a few NMIs recently. The method used single channel obtained low level of uncertainty with the phase-lock loop (PLL) synchronization, but the dual channel sampling method achieved high level of uncertainty. The one reason is that the sampled signal is not phase locked to the source signal $[1,2]$ or not used proper algorithm to compensate the amplitude and phase errors [5]. The precision measurement of alternating (ac) signals by digital sampling can be best accomplished with the use of a PLL and proper method was suggested in [5] with hardware modification in dual channel sampling method to synchronize the voltage and current signals. The other important parameter is root mean square (rms) measurements of the ac signal in the dual channel sampling technique. In this study more accurate than the one previously used in dual channel sampling technique method was used to determine the rms values with a low level of uncertainty comparing the algorithm with highly accurate thermal converters (TC) based AC/DC transfer calibration system This study mainly focused on the low level of measurement uncertainty with sophisticated algorithm which compensate the ratio and phase errors properly and a minimum of hardware development to approach level of uncertainty obtained with single channel sampling method. Although the proposed method operates at a power line frequency of $60 \mathrm{~Hz}$, it can be used as a reference standard within the frequency range of $20-100 \mathrm{~Hz}$ precisely.

\footnotetext{
* Correspondence: wije@kriss.re.kr
} 


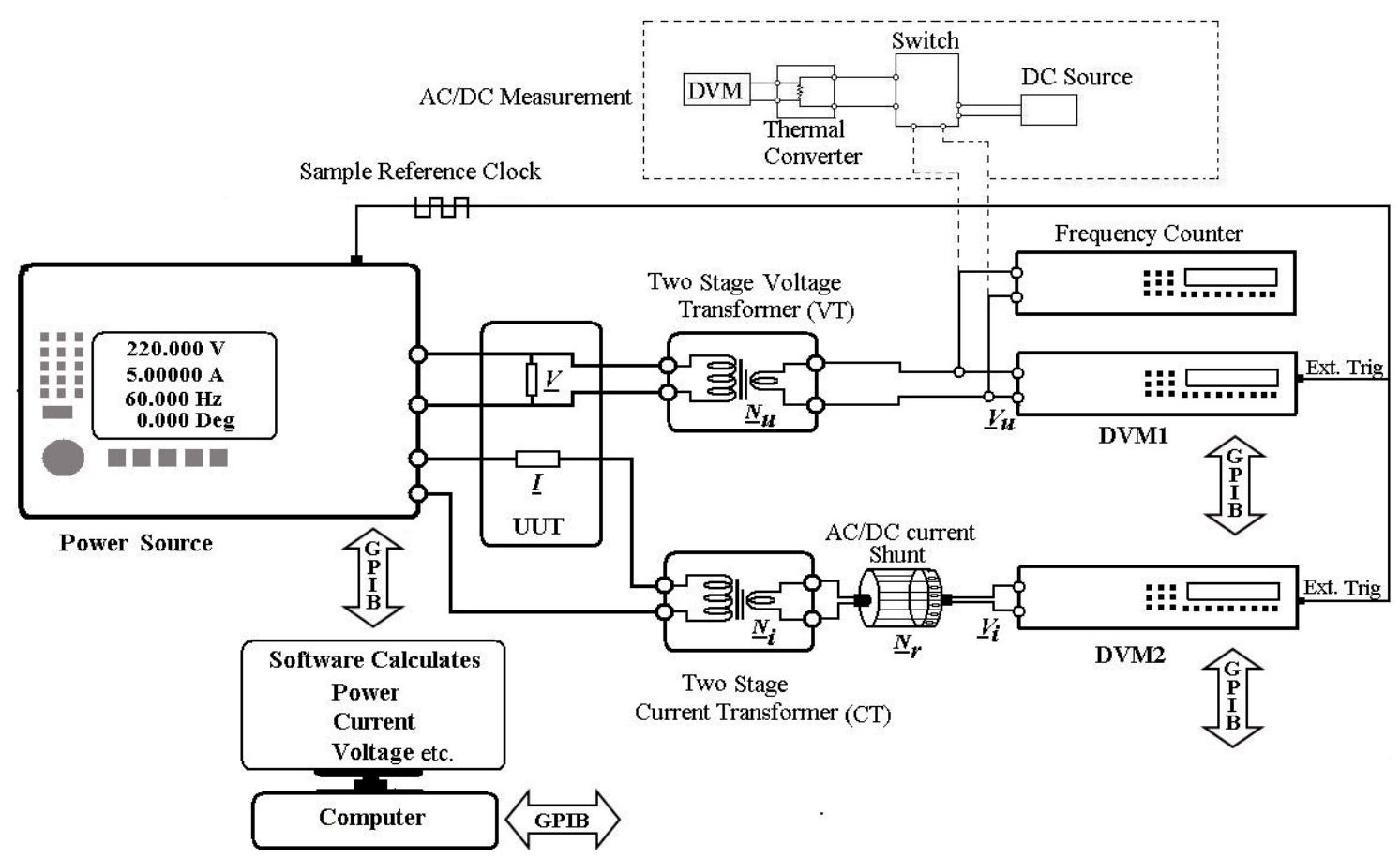

Fig. 1. Layout of the measuring system.

\section{Methods}

\subsection{The measuring system}

The measuring system of the reference power standard at KRISS is represented schematically in Figure 1. The system mainly consisted of a power source and two sampling DVMs measuring the outputs of the two-stage voltage transformer (VT) and the AC/DC current shunt. The current output $\underline{\boldsymbol{I}}$ was supplied to the unit under test (UUT) and also to the primary of the amplifier-aided two-stage current transformer (CT) up to $20 \mathrm{~A}$. The voltage output was supplied to the UUT and primary of the VT in parallel. The output of the CT was connected to the AC/DC current shunt, and the nominal values of the output voltages, $\underline{\boldsymbol{V}}_{\boldsymbol{u}}$ and $\underline{\boldsymbol{V}}_{\boldsymbol{i}}$, were $1 \mathrm{~V}$. Both voltages were connected to the sampling voltmeters directly. The two DVMs were operated as a waveform acquisition system, controlled by a computer with an IEEE 488 interface. All the components, $\mathrm{CT}$, voltage transformer (VT) and shunt, were designed in the laboratory.

The clock generated by the source, i.e., the data acquisition system, was controlled by the program and was coupled to the external trigger input of both DVMs. The sample and phase reference were provided by the source, Fluke 6100 A, and could be controlled by the GPIB commands. Figure 2 shows the relationship between the reference signal and the analog output. The sample reference appears only when the positive zero crossing of the phase reference signal in the GPIB is "ON" and triggers both DVMs to start sampling the waveforms data, phase locked to the master clock of the source. In this synchronization technique a time delay does not appear; however,

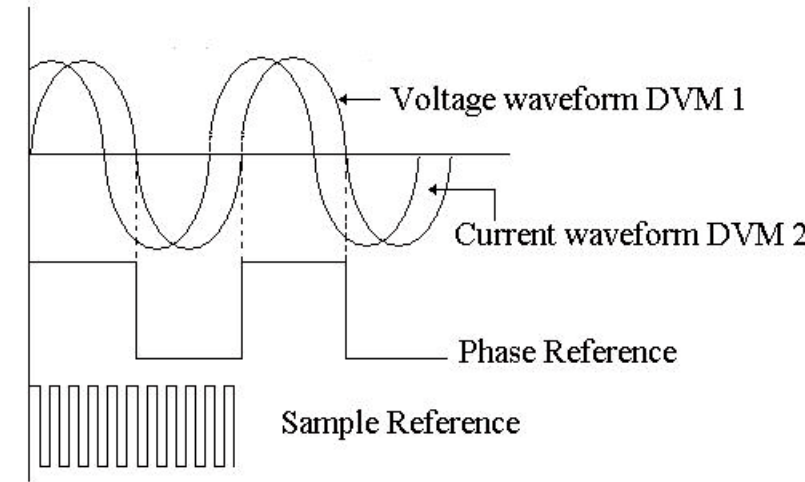

Fig. 2. Synchronization pattern of the DVMs.

a small jitter between two DVMs occurs and can be corrected through the program. The details will be discussed in Section 2.3. Both channels take 256 samples per cycle, yielding 2560 samples in 10 cycles, which are averaged to obtain good results. The frequency counter measures the frequency of the waveform and the measured value is used in the data processing algorithm.

\subsection{Ac voltage measurements}

Precise measurements of the low frequency ac voltage are needed for accurate power measurements. The most precise method to determine the rms value of an ac voltage is the AC/DC transfer with multi junction TCs. It is known that the $\mathrm{AC} / \mathrm{DC}$ transfer difference increases together with its uncertainty below $100 \mathrm{~Hz}$ and it can be evaluated with an uncertainty of $0.5 \mu \mathrm{V} / \mathrm{V}$ down to 
$10 \mathrm{~Hz}[6]$. The other approach to determine the rms value of the ac voltage is the sampling technique with an ADC. The precision of the sampling method depends on the choice of the ADC and usually a high precision DVM, HP-3458A, is used for this purpose. In the past, several laboratories initiated research and development of a sampling technique by using DVM, and one of the first important contributions was Swerlein's algorithm [7]. The data processing in the algorithm was in time domain and it has been thoroughly tested in reference [8]. Also, the extension version of Swerlein's algorithm [9] processed the sampling data in the frequency domain and measured the low frequency ac voltage within a level of uncertainty of $2.5 \mu \mathrm{V} / \mathrm{V}$. Therefore, a combined method was chosen to calculate the rms voltages, $V_{u}$, and $V_{i}$, which are coupled to the two DVMs operating in the DCV mode, in which the voltage signal is directly applied to the input of a high resolution integrating analog-to-digital converter (IADC).

For a total number of $N$ samples taken at a sampling time $T_{s}$, the discrete Fourier transform $D F T[k]$ for positive frequencies is defined as:

$$
D F T[k]=\frac{2}{N} \sum_{n=0}^{N-1} V\left(n T_{s}\right) e^{-j 2 \pi \cdot k \cdot\left(n T_{s}\right) / N}
$$

where $k=0,1, \ldots, N-1$

and $V\left(n T_{s}\right)$ is the time function of the input voltage, which depends on the sampling time $T_{s}$.

The Fast Fourier Transform function (FFT) was used to calculate DFT for each sample. The amplitudes of the DFTs need to be corrected further due to finite aperture $T_{a}$ (IADC frequency response correction), as expressed in [2], and this is:

$$
A_{k}=\frac{\sin \left(\pi k f T_{a}\right)}{\pi k f T_{a}}
$$

where $f$ is the fundamental frequency of the input signal and the rms value, $V_{r m s}$, of the input signal is given by:

$V_{r m s}=$

$E_{B W} E_{D C} \sqrt{V_{D C}^{2}+\frac{1}{2} \sum_{k=1}^{M} A_{k}\left(D F T \mathrm{Re}^{2}+D F T \mathrm{Im}^{2}\right)}$

where $M$ is the desired harmonics number, $V_{D C}$ is the $\mathrm{DC}$ component of the voltage signal, $E_{B W}$ is the finite bandwidth correction of the DVM and $E_{D C}$ is the correction of the DC voltage mode error.

The uncertainties of the calculated value mainly depend on uncertainties of the above correction factors. In addition, the following factors contribute to the level of uncertainty.

(a) DVM sampling noise;

(b) waveform digitization;

(c) trigger jitter;

(d) DVM analog input filter, etc.
However, the uncertainty calculation for the measured rms value using the above mentioned factors is somewhat complicated. Therefore, the algorithm calculated value directly compared with the $\mathrm{AC} / \mathrm{DC}$ measurement unit as shown in Figure 1. The nominal value of the voltage was $1 \mathrm{~V}$ and the system maintains this nominal value for different voltage $(\underline{\boldsymbol{V}})$ inputs. Therefore, the calibration results included the data processing algorithm and always compensate the corrections automatically before calculating the power parameters. This technique simplifies the operation and uncertainty calculations of the rms value. On the other hand, if we use a thermal converter (TC)based calibration system together with the proposed reference electrical power system to determine the $V_{u}$ with a low level of uncertainty as described in [4], it may create complication in the whole system and more sophisticated software would need to be developed.

\subsection{Determination of the electrical power}

The voltage $\underline{\boldsymbol{V}}$, current $\underline{\boldsymbol{I}}$ and the phase angle between them are quantities of interest to calculate active and reactive power. In the thermal power comparator, which utilizes the two TCs and summation amplifiers, a common mathematical expression is used to process the hardware calculation of power for easy design. Similarly, in the data processing software, the equation of the basic principle of power can be used, and it is:

$$
\underline{\boldsymbol{V}}_{\boldsymbol{u}} \cdot \underline{\boldsymbol{V}}_{\boldsymbol{i}}=\frac{1}{4}\left\{\left(\underline{\boldsymbol{V}}_{\boldsymbol{u}}+\underline{\boldsymbol{V}}_{\boldsymbol{i}}\right)^{2}-\left(\left(\underline{\boldsymbol{V}}_{\boldsymbol{u}}-\underline{\boldsymbol{V}}_{\boldsymbol{i}}\right)\right)^{2}\right\}
$$

The quantities $\underline{\boldsymbol{V}}_{\boldsymbol{u}}$ and $\underline{\boldsymbol{V}}_{\boldsymbol{i}}$ can be expressed in terms of $\underline{\boldsymbol{V}}$ and $\underline{\boldsymbol{I}}$ combining with the VT turn ratio $\underline{\boldsymbol{N}}_{\boldsymbol{u}}$, the CT turn ratio $\underline{N}_{i}$ and the AC/DC current shunt resistor impedance $\underline{\underline{N}}_{\boldsymbol{r}}$, and they are:

$$
\underline{V}=\underline{N}_{u} \cdot \underline{V}_{u}
$$

and

$$
\underline{I}=\underline{N}_{i} \cdot \frac{\underline{V}_{i}}{\underline{N}_{r}}
$$

After digitizing, the analog input voltages of the DVM may be expressed as complex numbers because the FFT algorithm calculated the real and imaginary parts of the complex DFTs for the fundamental and its harmonics. Therefore, $\underline{\boldsymbol{V}}_{\boldsymbol{u}}$ and $\underline{\boldsymbol{V}}_{\boldsymbol{i}}$ are complex voltages and may be written as:

$$
\underline{\boldsymbol{V}}_{\boldsymbol{i}}=C_{i}+j D_{i}
$$

and

$$
\underline{\boldsymbol{V}}_{\boldsymbol{u}}=C_{u}+j D_{u}
$$

where $C_{i}, C_{u}, D_{i}$ and $D_{u}$ are the DFTRe(k) and $D F T \operatorname{Im}(\mathrm{k})$ of the voltage and current signals. The VT, CT and shunt resistor parameters, $\underline{\boldsymbol{N}}_{\boldsymbol{u}}, \underline{\boldsymbol{N}}_{\boldsymbol{i}}$ and $\underline{\boldsymbol{N}}_{\boldsymbol{r}}$, can be expressed as complex numbers using nominal value, $\boldsymbol{N}_{\boldsymbol{n}}$, of transformation ratios or shunt resistance, ratio errors $(\alpha)$, and relative phase errors $(\beta)$. A common expression for the above parameters is given below:

$$
\underline{\boldsymbol{N}}_{u, i, r}=N_{n(u, i, r)}\left(1+\alpha_{u, i, r}+j \beta_{u, i, r}\right)
$$




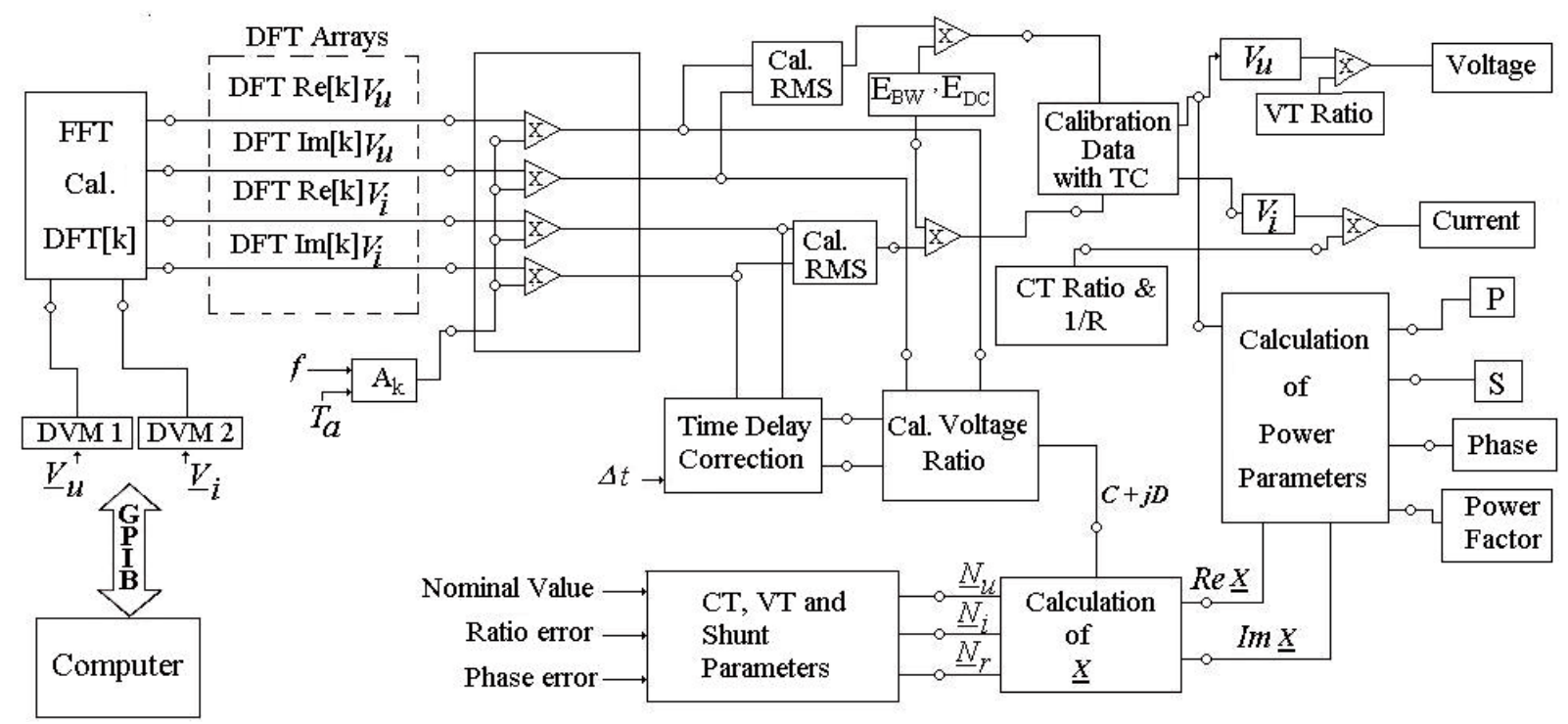

Fig. 3. Software algorithm.

Then, the main power parameters of the input signals can be calculated by combining equations (4) and (5), and may be written as follows:

The active power:

$$
P=\frac{N_{n u} N_{n i}}{N_{n r}} V_{u}^{2} \operatorname{Re} \underline{\boldsymbol{X}}
$$

and the apparent power:

$$
S=\frac{N_{n u} N_{n i}}{N_{n r}} V_{u}^{2}|\underline{X}|
$$

where $V_{u}$ is the rms value of the input voltage of DVM1 and $\underline{\boldsymbol{X}}$ is:

$$
\underline{\boldsymbol{X}}=\frac{\left(1+\alpha_{u}+j \beta_{u}\right)\left(1+\alpha_{i}+j \beta_{i}\right)\left(1+\alpha_{r}-j \beta_{r}\right)}{\left(1+\alpha_{r}\right)^{2}+\beta_{r}^{2}} \cdot \frac{\underline{\boldsymbol{V}}_{\boldsymbol{i}}}{\underline{\boldsymbol{V}}_{\boldsymbol{u}}}
$$

The real and imaginary components of the complex number $\underline{\boldsymbol{X}}$ are computed by combining equations (6) and (7).

\subsection{Algorithm}

Figure 3 shows the procedure for the development of data analysis software step by step using several subprograms. At first the digitized signals acquired from the two DVMs and FFT algorithm calculate the real and imaginary parts of the DFTs, which are stored in DFT arrays. The DFTs of the fundamental frequency and its harmonics up to the desired number were selected and multiplied by the frequency correction factor $A_{k}$. The subprogram of the rms calculation, CAL. RMS, calculates the values of the input signals according to equation (3) and then subject to the TC calibration data comparator which determines the rms values $V_{u}$ and $V_{i}$. Then, the voltage and current values are calculated using CT, VT and the shunt parameters, which are stored in a file. The time delay correction procedure reconstructs the DFTs of the current signal before calculating the complex voltage ratio to eliminate the phase error. The phase angle of the two signals is:

$$
\arctan \frac{D_{u}}{C_{u}}-\arctan \frac{D_{i}}{C_{i}}=\varphi
$$

The $\varphi=0 \mu \mathrm{rad}$ gives the best results and $C_{i} / D_{i}$ should be corrected to fulfill the requirement. Therefore, the real and imaginary DFTs of $\underline{\boldsymbol{V}}_{\boldsymbol{i}}$ were further corrected considering the time delay $(\Delta t)$ between two DVMs due to jitter, which includes a rotation of $2 \pi k f D$ rad [2] having a fundamental frequency $f$ in any harmonic component. The resulting values are:

$$
\begin{aligned}
& C_{i c}=C_{i} \cos (2 \pi k f \Delta t)-C_{i} \sin (2 \pi k f \Delta t) \\
& D_{i c}=D_{i} \cos (2 \pi k f \Delta t)+C_{i} \sin (2 \pi k f \Delta t)
\end{aligned}
$$

The $C_{i c}$ and $D_{i c}$ are determined by the program, and the corrected $\underline{\boldsymbol{V}}_{\boldsymbol{i}}$ is:

$$
\underline{\boldsymbol{V}}_{\boldsymbol{i}}=C_{i c}+j D_{i c}
$$

and the complex voltage ratio is:

$$
\begin{aligned}
\frac{\underline{\boldsymbol{V}}_{\boldsymbol{i}}}{\underline{\boldsymbol{V}}_{u}}=\frac{C_{i c}+j D_{i c}}{C_{u}+j D_{u}}= & \frac{C_{i c} C_{u}+D_{i c} D_{u}}{C_{u}^{2}+D_{u}^{2}} \\
& +\frac{C_{u} D_{i c}-C_{i c} D_{u}}{C_{u}^{2}+D_{u}^{2}} j=C+j D
\end{aligned}
$$

The phase error was eliminated according to the above calculation and we evaluated the system using the same voltage signal applied to both DVMs. By adjusting the time delay the average phase angle $\varphi \mu \mathrm{rad}$ minimized to nearly zero within $2 \mu \mathrm{rad}$.

By combining complex numbers, voltage ratio $\mathrm{CT}$, VT and shunt parameters, the program computes the vector 
$\underline{\boldsymbol{X}}$ and its real and imaginary components. As a final step the algorithm calculates power parameters and other parameters such as the power factor and phase angle. The program provides facilities to calibrate power meters and calibrators. A fully sophisticated algorithm was developed using LabView, a product of National Instruments, and all power parameters were calculated and display on the screen.

\section{Errors and uncertainties}

Amplitude and phase angle errors are introduced by several sources and affect the accuracy of power measurements. Those errors can be compensated through the program with a known level of uncertainty.

The correction factors mainly depend on $V_{u}$ and $\underline{\boldsymbol{X}}$, because the power parameters were calculated using equations (8) and (9). According to ISO GUM method [10] the uncertainty of the active power measurements can be written using Taylor series as follows:

$$
\begin{array}{r}
U^{2}(P)=\left(\frac{\partial P}{\partial V_{u}}\right)^{2} U^{2}\left(V_{u}\right)+\left(\frac{\partial P}{\partial \operatorname{Re} \underline{\boldsymbol{X}}}\right)^{2} U^{2}(\operatorname{Re} \underline{\boldsymbol{X}}) \\
+2\left(\frac{\partial P}{\partial V_{u}}\right)\left(\frac{\partial P}{\partial \operatorname{Re} \underline{\boldsymbol{X}}}\right) \operatorname{Cov}\left(V_{u}, \operatorname{Re} \underline{\boldsymbol{X}}\right)
\end{array}
$$

Equation (16) shows that the Active power measurements uncertainty mainly depends on voltage rms measurements, $V_{u}$, the term $\operatorname{Re} \underline{\boldsymbol{X}}$ and their correlation factor. The measurements are calculated by DFT estimation obtained from the digital sampling of the input waveforms. Therefore, the quantities $V_{u}$ and $\operatorname{Re} \underline{\boldsymbol{X}}$ were assumed to be correlated. As discussed in Section 2.3, $V_{u}$ is the rms value measured with DVM1. $V_{u}$ can be evaluated by a sampling technique and then calibrated using TC with a level of uncertainty of $1 \mu \mathrm{V} / \mathrm{V}$. The $\operatorname{Re} \underline{\boldsymbol{X}}$ derived from the equations (10) and (15).

The CT and VT are additional sources contributing to both phase angle and ratio errors in power calculation as shown in equation (10). The errors, applied as shown in equation (7), and the compensation procedures were included in the data processing algorithm. The levels of uncertainty were $0.5 \mu \mathrm{V} / \mathrm{V}, 0.5 \mu \mathrm{A} / \mathrm{A}$ and $2 \mu \mathrm{rad}$ for both $\mathrm{CT}$ and VT. The impedance of the shunt resistor $\left|\underline{\boldsymbol{N}}_{r}\right|$ was evaluated periodically and its value is known with an uncertainty of $0.5 \mu \Omega / \Omega$ and the phase error evaluated within $2 \mu \mathrm{rad}$. The uncertainty of the $\operatorname{Re} \underline{\boldsymbol{X}}$ is:

$$
\begin{aligned}
& U^{2}(\operatorname{Re} \underline{\boldsymbol{X}})= \\
& \left\{\begin{array}{l}
\left(\frac{\partial \operatorname{Re} \underline{\boldsymbol{X}}}{\partial \alpha_{i}}\right)^{2} U^{2}\left(\alpha_{i}\right)+\left(\frac{\partial \operatorname{Re} \underline{\boldsymbol{X}}}{\partial \alpha_{u}}\right)^{2} U^{2}\left(\alpha_{u}\right) \\
+\left(\frac{\partial \operatorname{Re} \underline{\boldsymbol{X}}}{\partial \alpha_{r}}\right)^{2} U^{2}\left(\alpha_{r}\right)+\left(\frac{\partial \operatorname{Re} \underline{\boldsymbol{X}}}{\partial \beta_{i}}\right)^{2} U^{2}\left(\beta_{i}\right) \\
+\left(\frac{\partial \operatorname{Re} \underline{\boldsymbol{X}}}{\partial \beta_{u}}\right)^{2} U^{2}\left(\beta_{u}\right)+\left(\frac{\partial \operatorname{Re} \underline{\boldsymbol{X}}}{\partial \beta_{r}}\right)^{2} U^{2}\left(\beta_{r}\right) \\
+\left(\frac{\partial \operatorname{Re} \underline{\boldsymbol{X}}}{\partial C}\right)^{2} U^{2}(C)+\left(\frac{\partial \operatorname{Re} \underline{\boldsymbol{X}}}{\partial D}\right)^{2} U^{2}(D)
\end{array}\right\}
\end{aligned}
$$

The uncertainties of relative ratio errors $U^{2}(\alpha)$ and phase errors $U^{2}(\beta)$ are known as mentioned above and considered as normal distribution functions. The sensitivity coefficients of each parameter were computed separately assuming multiplications of $\alpha, \beta$ and $\alpha \beta$ approximately zero since those factors are in the order of $10^{-6}$. The coefficients of $C$ and $D$ are always 1 and zero respectively and the others equal to the values of $C$ and $D$ which depend on the phase angle of voltage and current waveforms. The calculated values are shown in Table 1 . The uncertainties of $C$ and $D$ and the other quantities should be known in equation (17).

The $C$ and D, DFT ratios, in equation (15) are the real and imaginary components of the complex voltage ratio. The uncertainties of the DFT components depend on the DVM characteristics and FFT computation error. The DVM characteristics mentioned in Section 2.2 affect to the DFT amplitude uncertainty. Considering these factors the uncertainty can be determined analytically as mentioned in [4] or can be evaluated experimentally. In this study we used experimental method to evaluate the DFT ratio and DFT amplitude errors. In Section 2.2, it was shown that rms voltage value calculated using DFTs and compared with TC was in agreement within $1 \mu \mathrm{V} / \mathrm{V}$. Therefore, maximum value of the DFT calculation uncertainty can be assumed as normal distribution function with the value of $\sqrt{2} \mu \mathrm{V} / \mathrm{V}$. The TC calibration uncertainty $1 \mu \mathrm{V} / \mathrm{V}$ also includes the assumed value. Therefore the DFT calculation uncertainty is less than $1 \mu \mathrm{V} / \mathrm{V}$. Since the $D F T$ uncertainty is known the $U^{2}(C)$ and $U^{2}(D)$ in equation (17) were computed applying Taylor series to equation (15). The calculated value for complex voltage ratio uncertainty for any phase angle is $1 \times 10^{-6}$. The final term in equation (16) which has to be known is correlation coefficient to calculate uncertainty of active power measurements. The covariance factor between $V_{u}$ and $\operatorname{Re} \underline{\boldsymbol{X}}$ may be written as follows:

$$
\operatorname{Cov}\left(V_{u}, \operatorname{Re} \underline{\boldsymbol{X}}\right)=\left\{\begin{array}{l}
\frac{\partial V_{u}}{\partial C_{u}} \frac{\partial \operatorname{Re} \underline{\boldsymbol{X}}}{\partial C_{u}} U^{2}\left(C_{u}\right) \\
+\frac{\partial V_{u}}{\partial D_{u}} \frac{\partial \operatorname{Re} \underline{\boldsymbol{X}}}{\partial D_{u}} U^{2}\left(D_{u}\right)
\end{array}\right\}
$$

The coefficients were calculated for different phase angles and values were summarized in Table 1 . In addition to the factors mentioned above in equation (16), the other common factors were considered for uncertainty budget. The first one is the phase difference, $U_{p h}$, between two DVMs and the error, $2 \mu \mathrm{rad}$, contributes $2 / \sqrt{3} \quad \mu \mathrm{W} / \mathrm{VA}$ as rectangular distribution functions. Second one is short term stability of the source, $U_{s}$, which has also rectangular distribution contributes $1 \mu \mathrm{W} / \mathrm{VA}$. The third is the short term drift of the DVMs and calculated uncertainty, $U_{d}$, is $0.5 \mu \mathrm{W} / \mathrm{VA}$. The repeatability of the measurement uncertainty, $U_{A}$, contributes $1 \mu \mathrm{W} / \mathrm{VA}$. All the values mentioned above are calculated for the voltage $220 \mathrm{~V}$ and current $5 \mathrm{~A}$. The nominal ratios, $N_{n u}$ and $N_{n i}$ of the VT and CT are 220 and 50 respectively. The nominal value of the shunt resistor, $N_{n r}$, is $10 \Omega$. The calculation was carried out for different power factors (PF) including $0,0.7$ and 1 as shown in Table 1. 
Table 1. Summary of the uncertainty budget.

\begin{tabular}{|c|c|c|c|}
\hline \multirow{2}{*}{ Parameter } & \multicolumn{3}{|c|}{ Phase angle $(\varphi)$} \\
\hline & $\varphi=0^{0}$ & $\varphi=45^{0}$ & $\varphi=90^{\circ}$ \\
\hline Voltage ratio & $C=1$ & $C=0.707$ & $C=0$ \\
\hline Coefficients & $D=0$ & $D=-0.707$ & $D=-1$ \\
\hline$\left(\frac{\partial \operatorname{Re} \underline{\boldsymbol{X}}}{\partial \alpha}\right)_{u, i, r}=$ & 1 & 0.707 & 0 \\
\hline$\left(\frac{\partial \operatorname{Re} \underline{\boldsymbol{X}}}{\partial \beta}\right)_{u, i, r}=$ & 0 & -0.707 & -1 \\
\hline$\left(\frac{\partial \operatorname{Re} \underline{\boldsymbol{X}}}{\partial C}\right)=$ & 1 & 1 & 1 \\
\hline$\left(\frac{\partial \operatorname{Re} \underline{\boldsymbol{X}}}{\partial D}\right)=$ & 0 & 0 & 0 \\
\hline$U^{2}(\operatorname{Re} \underline{\boldsymbol{X}})=$ & $1.735 \times 10^{-6}$ & $7.375 \times 10^{-6}$ & $13 \times 10^{-6}$ \\
\hline$\left(\frac{\partial P}{\partial V_{u}}\right)=$ & $2 \times 1100$ & $1.4 \times 1100$ & 0 \\
\hline$\left(\frac{\partial P}{\partial \operatorname{Re} \underline{\boldsymbol{X}}}\right)=$ & 1100 & 1100 & 1100 \\
\hline $\operatorname{Cov}\left(\overline{V_{u}}, \operatorname{Re} \underline{\boldsymbol{X}}\right)=$ & $-0.5 \times 10^{-6}$ & $-0.354 \times 10^{-6}$ & 0 \\
\hline$\frac{U^{2}(P)}{(V A)^{2}}=$ & $3.8(\mu \mathrm{W} / \mathrm{VA})^{2}$ & $9.4(\mu \mathrm{W} / \mathrm{VA})^{2}$ & $13(\mu \mathrm{W} / \mathrm{VA})^{2}$ \\
\hline $\begin{array}{l}\text { Common } \\
\text { parameters }\end{array}$ & $\begin{array}{l}U_{d}=0.5 \mu \mathrm{W} / \\
U_{p h}=2 / \sqrt{3}\end{array}$ & $\begin{array}{l}U_{s}=1 \mu \mathrm{W} / \mathrm{VA}, \\
\mathrm{VA}, U_{A}=1 \mu \mathrm{W}\end{array}$ & \\
\hline $\begin{array}{l}\text { Combined standard } \\
\text { uncertainty }\end{array}$ & $\begin{array}{l}2.7 \mu \mathrm{W} / \mathrm{VA}(\varphi \\
3.6 \mu \mathrm{W} / \mathrm{VA}(\varphi \\
4.1 \mu \mathrm{W} / \mathrm{VA}(\varphi\end{array}$ & $\begin{array}{l} \\
\left.5^{0}\right) \\
\left.0^{0}\right)\end{array}$ & \\
\hline
\end{tabular}

Table 2. Calibration results of the test power meter (RD 33).

\begin{tabular}{lcccccc}
\hline \multirow{2}{*}{ Nominal value } & \multicolumn{2}{c}{ PF=1 } & \multicolumn{2}{c}{ PF $=0.5$ lead } & \multicolumn{2}{c}{ PF $=0.5$ lag } \\
\cline { 2 - 7 } & Ref. Std. (W) & UUT (W) & Ref. Std. (W) & UUT (W) & Ref. Std. (W) & UUT (W) \\
\hline $120 \mathrm{~V} / 1$ A & 120.01879 & 120.0248 & 60.00718 & 60.0123 & 60.00815 & 60.0113 \\
$120 \mathrm{~V} / 5 \mathrm{~A}$ & 599.82996 & 599.8601 & 299.91378 & 299.9325 & 299.91795 & 299.9314 \\
$220 \mathrm{~V} / 1 \mathrm{~A}$ & 220.03311 & 220.0446 & 110.01438 & 110.0232 & 110.01616 & 110.0206 \\
$220 \mathrm{~V} / 5 \mathrm{~A}$ & 1099.6982 & 1099.7489 & 549.83710 & 549.8862 & 549.84569 & 549.8735 \\
\hline
\end{tabular}

\section{Results and discussion}

A detailed uncertainty calculation for different power factors shows that the developed system has a level of uncertainty less than $4 \mu \mathrm{W} / \mathrm{VA}$ for any power factor. The coverage factor mainly depends on the type of distribution function and the major contribution, normal distribution function, in the uncertainty budget. Therefore, it was assumed that the coverage factor $k=2$ with the $95 \%$ of confidence level. The main components of the uncertainty analysis are the calibration uncertainties of the CT, VT and current shunt, $\mathrm{AC} / \mathrm{DC}$ transfer uncertainty of the rms measurement and phase error of the system. As mentioned in Section 1, the demands for the traceable electrical power calibration have grown up in the last few years rapidly with the low level of calibration uncertainty. After completion of the reference (Ref. Std.) power measuring system many types of power meters have been calibrated within the laboratory according to the industrial demand. The RD33 product of Radian Co., one common power meter needs traceable calibration with low level of uncertainty.
The meter also uses as a secondary standard in the laboratory to calibrate other power meters and power sources as request of the industrial laboratories. For example Table 2 shows the calibration results of the power meter used as UUT in the system. The input voltages are $120 \mathrm{~V}$ and $220 \mathrm{~V}$. The current range is $1 \mathrm{~A}$ to $5 \mathrm{~A}$. The calibrations were carried out for the power factors $1,0.5$ lead and 0.5 lag. Table 3 shows the calibration results of another common power meter, MSB 100 from Rotek Co., uses in the industrial power calibration. According to the results the errors were found to be within $50 \mu \mathrm{W} / \mathrm{VA}$ and $100 \mu \mathrm{W} / \mathrm{VA}$ respectively. The calibration uncertainty of both examples depends on repeatability of the test meters, $2 \mu \mathrm{W} / \mathrm{VA}$, and the uncertainty of the reference standard. Therefore, the calibration uncertainty of the determined errors of the meters was $10 \mu \mathrm{W} / \mathrm{VA}$ with the $95 \%$ of confidence level.

The derived uncertainty for the reference system including all possible contribution as shown in Table 1 is higher than the single channel sampling technique used 
Table 3. Calibration results of the test power meter (MSB 100).

\begin{tabular}{lcccccc}
\hline \multirow{2}{*}{ Nominal value } & \multicolumn{2}{c}{ PF=1 } & \multicolumn{2}{c}{ PF $=0.5$ lead } & \multicolumn{2}{c}{ PF $=0.5$ lag } \\
\cline { 2 - 7 } & Ref. Std. (W) & UUT (W) & Ref. Std. (W) & UUT (W) & Ref. Std. (W) & UUT (W) \\
\hline $120 \mathrm{~V} / 1$ A & 120.01603 & 120.0047 & 60.00804 & 60.0019 & 60.00922 & 60.0025 \\
$120 \mathrm{~V} / 5$ A & 599.8401 & 599.782 & 299.9052 & 299.880 & 299.9184 & 299.890 \\
$220 \mathrm{~V} / 1$ A & 220.03064 & 220.0087 & 110.01284 & 110.0020 & 110.01588 & 110.0058 \\
$220 \mathrm{~V} / 5$ A & 1099.6999 & 1099.591 & 549.8485 & 549.796 & 549.8567 & 549.802 \\
\hline
\end{tabular}

in [3] and [4]. With improvement in VT and CT accuracy and their calibration uncertainties, the level of uncertainty of the system is bound to be significantly reduced. Although the uncertainty due to phase jitter, the main difference of single and dual channel sampling methods, between two DVMs is unavoidable from the system the level of uncertainty $(k=1)$ within $2 \mu \mathrm{W} / \mathrm{VA}$ will be obtained without a special hardware modification.

\section{Conclusion}

The application of a sampling technique that utilizes DVMs has allowed the design of a system for the measurement of electrical power for power frequencies having relative uncertainties lower than $4 \mu \mathrm{W} / \mathrm{VA}(k=1)$ for any power factor. The proper algorithms and techniques used in the system allowed to improve the uncertainty of the dual channel sampling method and the uncertainty is very close to the single channel method. The system is now used at KRISS as a primary standard for the precision measurement of electrical power to fulfill the industrial demand.

\section{References}

1. E. Toth, A.M.R. Franco, R.M. Debatin, Power and energy reference system, applying dual-channel sampling, IEEE Trans. Instrum. Meas. 54, 404 (2005)
2. U. Pogliano, Use of integrative analog-to-digital converters for high precision measurement of electric power, IEEE Trans. Instrum. Meas. 50, 1315 (2001)

3. G. Rammn, H. Moser, Andreas, A New scheme for Generating and Measuring Active, Reactive and Apparent Power at Power Frequencies with Uncertainties of $2.5 \times$ $10^{-6}$, IEEE Trans. Instrum. Meas. 48, 422 (1999)

4. W.G.K. Ihlenfeld, E. Mohns, K. Dauke, Classical Nonquantum AC Power Measurements With Uncertainties Approaching $1 \mu \mathrm{W} / \mathrm{VA}$, IEEE Trans. Instrum. Meas. 56, $410(2007)$

5. S. Svensson, K.-E. Rydler, A measuring system for the calibration of power analyzers, IEEE Trans. Instrum. Meas. 44, 316 (1995)

6. M. Klonz, AC-DC transfer difference of the PTB multijunction thermal converter in the frequency range from 10 Hz to 100 kHz, IEEE Trans. Instrum. Meas. 36, 320 (1987)

7. R.L. Swerlein, A 10 ppm accurate digital ac measurement algorithm (Hewlett-Packard internal publication, 1991)

8. M. Kampik, H. Laiz, M. Klonz, Comparison of three accurate methods to measure $\mathrm{AC}$ voltage at low frequencies, IEEE Trans. Instrum. Meas. 49, 429 (2000)

9. G.A. Kyriazis, Extension of Swerlein's algorithm for AC voltage measurement in the frequency domain, IEEE Trans. Instrum. Meas. 52, 367 (2003)

10. Guide to the expression of uncertainty in measurement, first edn. (International Organisation for Standardization, Switzerland, 1993) 